\title{
多重連結型移動ビークルの軌道安定化制御のための簡易設計法
}

\author{
正員田中一 男 (電気通信大学) \\ 非会員堀強 (電気通信大学) \\ 非会員 岩 崎 正 明 (電気通信大学)
}

\section{Trajectory Control of a Vehicle with Triple Trailers}

\begin{abstract}
Kazuo Tanaka, Member, Tsuyoshi Hori, Non-member, Masaaki Iwasaki, Non-member (The University of Electro-Communications)
\end{abstract}

We consider the backing up control of a vehicle with triple trailers via a model-based fuzzy control methodology. To begin with, the vehicle dynamics is represented by a Takagi-Sugeno fuzzy model which is equivalent to a simplified nonlinear model of the vehicle. Then we employ the so-called "parallel distributed compensation" design to arrive at a controller that guarantees the stability of the closed-loop system consisted of the fuzzy model and controller. The control design problem is cast in terms of linear matrix inequalities. The simulation and experimental results demonstrate that the fuzzy controller effectively achieves the backing up control of the vehicle with triple trailers.

キーワード：多重連結型移動ビータル, ファジィ制御, 並列分散的補償, 安定性、線形行列不等式

\section{1.まえがき}

トレーラ・トラック型移動ビークル（以下，多重連結型 移動ビークルと呼ぶ）の後退制御は非常に困難な非線形制 御問題であり、これを夷現するためにはジャックナイフ現 象を避ける必要がある。この問題に対しては様々な解法が 提案されてきた(1)-(8)。しかし、文献 $(1)$ 亿 (4)ではシミュ レーションのみの報告にもかかわらず，制御系の安定性も 解析されていない。1台トレーラの場合について安定な制御 器を設計し，モデル機を作製し実験が行われている(5)(6)。 また，文献(7)，(8)に扔いては 2 台のトレーラを連結させ たモデル機の実験が行われている。一方，文献(5)の方法 を拡張し 2 台のトレーラを連結させたモデル㙨の実験も報 告されている(9)。文献 (9)では，安定なフィードバックゲ インが安定条件に基づく試行錯誤的な方法により求められ ていた。本論文では 2 台卜レーラよりも困難である 3 台卜 レーラの後退制卸を扱う。文献 (5),(9)による著者らの経 験では, トレーラの台数が増えるとその制御が極端に難し くなるので，本論文で扱う 3 台トレーラでは作製したモデ ル機での実験を成功させることを第一の目的とし，この目 的を達成するためにできるだ簡単に制御系の設計を行う ことを目指す。ただし，制御系の重要な概念である安定性 については（近似的にでも）その保証を行う。制御系の安 定性を解析するにあたり，本論文では著者らが導出した安 定条件(10)-(14)をさらに緩めた新しい安定条件を導出しこ
の安定条件の特別な場合が従来の安定条件に相当すること を示す。この安定条件を利用して試行錯誤的なアプローチ を経ることなく直接安定なフィードバックゲインを求める。 新たに導出した安定条件に基づく設計法を本对象に適用し， シミュレーションと実験を通して本アプローチの有効性を 示す。

\section{2. ファジィモデル化}

図1に3台トレーラ(セミトレーラタイプ:ジョイントが 前の車両の後輪の中点にある夕イプ) のモデルとその座標 系を示す。本論文では以下のような非線形モデルを用いる。

$$
\begin{aligned}
x_{0}(t+1) & =x_{0}(t)+\frac{\nu \cdot \Delta t}{l} \tan (u(t)) \ldots \ldots \ldots \\
x_{1}(t) & =x_{0}(t)-x_{2}(t) \ldots \ldots \ldots \ldots \ldots \ldots \\
x_{2}(t+1) & =x_{2}(t)+\frac{\nu \cdot \Delta t}{L} \sin \left(x_{1}(t)\right) \ldots \ldots \ldots \\
x_{3}(t) & =x_{2}(t)-x_{4}(t) \ldots \ldots \ldots \ldots \ldots \\
x_{4}(t+1)= & x_{4}(t)+\frac{\nu \cdot \Delta t}{L} \sin \left(x_{3}(t)\right) \ldots \ldots \ldots \\
x_{5}(t)= & x_{4}(t)-x_{6}(t) \ldots \ldots \ldots \ldots \\
x_{6}(t+1)= & x_{6}(t)+\frac{\nu \cdot \Delta t}{L} \sin \left(x_{5}(t)\right) \ldots \ldots \ldots \\
x_{7}(t+1)= & x_{7}(t)+\nu \cdot \Delta t \cos \left(x_{5}(t)\right) \\
& \times \sin \left(\frac{x_{6}(t+1)+x_{6}(t)}{2}\right) \ldots \ldots
\end{aligned}
$$




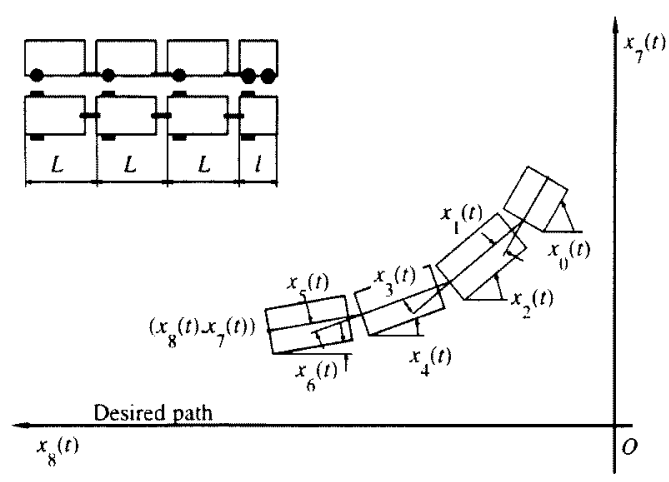

図 1 モデルと座標系

Fig. 1. Vehicle model with triple trailers.

$$
\begin{aligned}
x_{8}(t+1)= & x_{8}(t)+\nu \cdot \Delta t \cos \left(x_{5}(t)\right) \\
& \times \cos \left(\frac{x_{6}(t+1)+x_{6}(t)}{2}\right) .
\end{aligned}
$$

ここで, $l(=0.087[\mathrm{~m}])$ はトラック部の長さ, $L(=0.130[\mathrm{~m}])$ はトレーラ部の長さ， $\nu(=-0.10[\mathrm{~m} / \mathrm{sec}]$.$) は徯退速度，$ $\Delta t(=0.5[\mathrm{sec}]$.$) はサンプリング時間である。なお，モデル$ の記述において，たとえば (1) 式の左辺は $x(t+\Delta t)$ と 書くべきであるが、本論文では簡単のため $x(t+1)$ のよ うに記述する。 $x_{1}(t), x_{3}(t), x_{5}(t)$ のいずれかが $90[\mathrm{deg}$. あるいは $-90[$ deg.]になるとジャックナイフ現象を引き 起こすため，数多くのジャックナイフポジションが存在 する難しい系である。制御目的は前進することなく捘退 だけで直線 $\left(x_{7}=0\right)$ に沿わせることである。つまり， $x_{1}(t) \rightarrow 0, x_{3}(t) \rightarrow 0, x_{5}(t) \rightarrow 0, x_{6}(t) \rightarrow 0, x_{7}(t) \rightarrow 0$ を制御目的とする。

(1)－(8) 式を(10) 式の高木・菅野のファジィモデル (15) に䈯き換える。

Rule 1 :

$$
\begin{aligned}
& \text { If } p(t) \text { is "about } 0 \text { [rad.]", } \\
& \text { then } \boldsymbol{x}(t+1)=\boldsymbol{A}_{1} \boldsymbol{x}(t)+\boldsymbol{B}_{1} u(t)
\end{aligned}
$$

Rule 2:

$$
\text { If } p(t) \text { is "about } \pi \text { [rad.] or }-\pi \text { [rad.]", }
$$
then $\boldsymbol{x}(t+1)=\boldsymbol{A}_{2} \boldsymbol{x}(t)+\boldsymbol{B}_{2} u(t)$

ここで

$$
\begin{aligned}
& p(t)=x_{6}(t)+\frac{\nu \cdot \Delta t}{2 L} x_{5}(t), \\
& x(t)=\left[\begin{array}{llll}
x_{1}(t) & x_{3}(t) x_{5}(t) x_{6}(t) x_{7}(t)
\end{array}\right]^{T},
\end{aligned}
$$$$
A_{1}=\left[\begin{array}{ccccc}
1-\frac{\nu \cdot \Delta t}{L} & 0 & 0 & 0 & 0 \\
\frac{\nu \cdot \Delta t}{L} & 1-\frac{\nu \cdot \Delta t}{L} & 0 & 0 & 0 \\
0 & \frac{\nu \cdot \Delta t}{L} & 1-\frac{\nu \cdot \Delta t}{L} & 0 & 0 \\
0 & 0 & \frac{\nu \cdot \Delta t}{L} & 1 & 0 \\
0 & 0 & \frac{(\nu \cdot \Delta t)^{2}}{2 L} & \nu \cdot \Delta t & 1
\end{array}\right],
$$

$$
\begin{aligned}
& A_{2}=\left[\begin{array}{ccccc}
1-\frac{\nu \cdot \Delta t}{L} & 0 & 0 & 0 & 0 \\
\frac{\nu \cdot \Delta t}{L} & 1-\frac{\nu \cdot \Delta t}{L} & 0 & 0 & 0 \\
0 & \frac{\nu \cdot \Delta t}{L} & 1-\frac{\nu \cdot \Delta t}{L} & 0 & 0 \\
0 & 0 & \frac{\nu \cdot \Delta t}{L} & 1 & 0 \\
0 & 0 & \frac{g \cdot(\nu \cdot \Delta t)^{2}}{2 L} & g \cdot \nu \cdot \Delta t & 1
\end{array}\right], \\
& B_{1}=B_{2}=\left[\begin{array}{c}
\frac{\nu \cdot \Delta t}{\ell} \\
0 \\
0 \\
0
\end{array}\right]
\end{aligned}
$$

である。”about 0 [rad.]"および "about $\pi$ [rad.] or $-\pi$ [rad.]"は前件部のフォジィ集合であり，そのメンバーシッ プ関数は図 2のように求まる。このモデルの構築おいて， $u(t), x_{1}(t), x_{3}(t), x_{5}(t)$ が小さい值であると仮定した。モ デルの導出過程の詳細は付録に示寸。非ファジィ化後のファ ジィモデルは次のように表現できる。

$\boldsymbol{x}(t+1)=\frac{\sum_{i=1}^{2} w_{i}(p(t))\left\{\boldsymbol{A}_{i} \boldsymbol{x}(t)+\boldsymbol{B}_{i} u(t)\right\}}{\sum_{i=1}^{2} w_{i}(p(t))} \cdots$

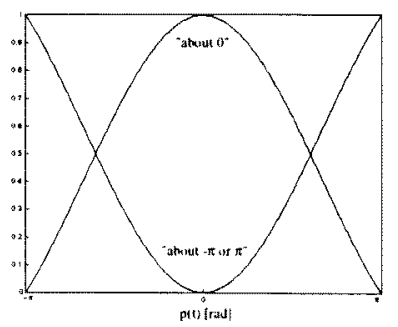

図 2 メンバーシップ関数

Fig. 2. Membership functions.

\section{3. 並列分散的補僧と安定条件}

〈3.1〉安定条件 （11）式の自由系は次の上うに表現 できる

$$
\boldsymbol{x}(t+1)=\sum_{i=1}^{r} h_{i}(p(t)) \boldsymbol{A}_{i} \boldsymbol{x}(t)(12)
$$

ここで, $r=2$ であり

$$
h_{i}(p(t))=\frac{w_{i}(p(t))}{\sum_{i=1}^{r} w_{i}(p(t))}
$$

である。(12) 式の安定性を保証する安定条件は Lyapunov の安定論のアプローチにより得られている。

\section{〔定理 1〕(10)(11) （13）式を満なす共通な正定対称行列 $P$ が存在すれば (12) 式で表わされるファジィシステムは大 域的に漸近安定である。}

$$
\boldsymbol{A}_{i}^{T} \boldsymbol{P} \boldsymbol{A}_{i}-\boldsymbol{P}<\mathbf{o}
$$


(12) 式のシステムにおいてはそれぞれのサブシステムが 安定, すなわちすべての $A_{i}$ が安定ならばシステム全体が安 定のように思えるが，これは一般には成り立なない。(11)(12)

〈3.2〉 並列分散的補傥によるファジィ制御器設計 次 に(10)式のファジィモデルに対してファジィ制御器を設計 する。並列分散的補償 (PDC) (11)-(14)によりファジイ制御 器を構成する。PDCによる制御器設計ではファジィモデル の 1 つ規則に対してそれを補償するようにファジィ制御 器の 1 つの規則を作成する。すなわち，(10) 式の Rule 1, 2 に対して Control Rule 1，2 作成する。

Control rule 1 :

If $p(t)$ is "about 0 [rad.]", then $u(t)=-\boldsymbol{F}_{1} \boldsymbol{x}(t)$

Control rule 2 :

If $p(t)$ is "about $\pi$ [rad.] or $-\pi$ [rad.]", then $u(t)=-\boldsymbol{F}_{2} \boldsymbol{x}(t)$

(14) 式のファジィ制䇝器は次のように表現できる。

$$
u(t)=-\sum_{i=1}^{2} h_{i}(p(t)) \boldsymbol{F}_{i} \boldsymbol{x}(t)
$$

ファジイ制御器設計問題は後件部のフィードバックゲイン $\boldsymbol{F}_{i}$ を決めることである。重要な点は, 制御器の構造はモデ ルのローカルな構造に着目して決定されているが, フィ一 ドバックゲイン $\boldsymbol{F}_{i}$ はグローバルな安定性（制御系全体の安 定性）を保証するように決定しなければならない。その理 由は，先に述べたようにローカルな安定性の保証（各サブ システムが安定) はグローパルな安定性を保証するとは限 らないからである。

(15) 式を(11) 式に代入すると (16) 式を得る。

$$
\begin{aligned}
\boldsymbol{x}(t+1)= & \sum_{i=1}^{r} \sum_{j=1}^{r} h_{i}(p(t)) h_{j}(p(t)) \boldsymbol{G}_{i j} \boldsymbol{x}(t) \\
= & \sum_{i=1}^{r} h_{i}^{2}(p(t)) \boldsymbol{G}_{i i} \boldsymbol{x}(t) \\
& +2 \sum_{i=1}^{r} \sum_{i<j} h_{i}(p(t)) h_{j}(p(t)) \\
& \times\left(\frac{\boldsymbol{G}_{i j}+\boldsymbol{G}_{j i}}{2}\right) \boldsymbol{x}(t) \ldots \ldots .
\end{aligned}
$$

ここで $r=2 ， \boldsymbol{G}_{i j}=\boldsymbol{A}_{i}-\boldsymbol{B}_{i} \boldsymbol{F}_{j}$ である，(16) 式にLyapunovの安定論を適用すると次の閉ループ系の安定条件が 得られる。

〔定理 2〕(1や) $s$ は制御中に同時に発火する規則数の最大 值であり，1 $\leq s \leq r$ であ。 $\boldsymbol{X}>\mathbf{o}, \boldsymbol{Y} \geq 0$ と (17)， (18) 式を棡たす $M_{i}(i=1,2)$ が存在すれば, (16) 式で 表わされる制御系は大域的漸近安定である。

$$
\left[\begin{array}{ll}
\boldsymbol{X}-(s-1) \boldsymbol{Y} & * \\
\boldsymbol{A}_{i} \boldsymbol{X}-\boldsymbol{B}_{i} \boldsymbol{M}_{i} & \boldsymbol{X}
\end{array}\right]>0 \quad \forall i
$$

$$
\begin{aligned}
& {\left[\begin{array}{lr}
\boldsymbol{X}+\boldsymbol{Y} & * \\
\frac{1}{2}\left\{\boldsymbol{A}_{i} \boldsymbol{X}+\boldsymbol{A}_{j} \boldsymbol{X}-\boldsymbol{B}_{i} \boldsymbol{M}_{j}-\boldsymbol{B}_{j} \boldsymbol{M}_{i}\right\} & \boldsymbol{X}
\end{array}\right]>0} \\
& i<j \text { s.t. } h_{i}(p(t)) h_{j}(p(t)) \neq 0
\end{aligned}
$$

ここで $M_{i}=F_{i} \boldsymbol{X}$ である。また* *は対称要素の転置行 列を表わす。

上記の条件は变数 $\boldsymbol{X}, \boldsymbol{Y}$ と $\boldsymbol{M}_{i}$ からなるLMI である。 LMI に基づく解法(16)(17)により，LMI 条件を満たす正定対 称行列 $\boldsymbol{X}$, 準正定対称行列 $\boldsymbol{Y}$ 及び行列 $\boldsymbol{M}_{i}$ が存在するな らば必ず見つけ出すことができる。フィードバックゲイン $F_{i}$ は $F_{i}=M_{i} X^{-1}$ で得られる。定理 2 はファジィ规則の 同時発火最大数 $\mathrm{s}$ とメンバーシップ関数の重なりを考慮し た条件となっており，一般的なポリトープ型よりシャープ な条件となっている。4章ではさらに緩い条件を導出する。 共通な $\boldsymbol{B}\left(\boldsymbol{B}_{1}=\boldsymbol{B}_{2}=\cdots=\boldsymbol{B}_{r}\right)$ 行列の場合には定理 2 は簡単になる(9)(13)(14)。(10) 式のファジィモデルは共通の $\boldsymbol{B}$ 行列を持つが本論文では共通の $\boldsymbol{B}$ 行列の場合ではなく一 般的な設計条件を与える。

\section{4. 緩い安定条件の導出}

本章では緩い安定条件を導出する。

〔定理 3〕(19),(20) 式の条件を满たす正定対称行列 $X$, 行 列 $M_{i}(\mathrm{i}=1, \cdots, \mathrm{r})$ および $\boldsymbol{T}_{i j}=\boldsymbol{T}_{j i}$ が存在すれば (16) 式 で表される制御系は大域的渐近安定である。フィードバッ クゲインはこの LMI の解 $\boldsymbol{X}, \boldsymbol{M}_{i}$ から $\boldsymbol{F}_{i}=\boldsymbol{M}_{i} \boldsymbol{X}^{-1}$ と して求めることができる。

$$
\begin{gathered}
{\left[\begin{array}{cc}
\boldsymbol{X}+\boldsymbol{T}_{i j} & * \\
\frac{1}{2}\left\{\boldsymbol{A}_{i} \boldsymbol{X}-\boldsymbol{B}_{i} \boldsymbol{M}_{j}+\boldsymbol{A}_{j} \boldsymbol{X}-\boldsymbol{B}_{j} \boldsymbol{M}_{i}\right\} & \boldsymbol{X}
\end{array}\right]>\mathbf{0}} \\
i \leq j \text { s.t. } h_{i}(p(t)) \cap h_{j}(p(t)) \neq 0 \cdots \cdots(19) \\
T=\left[\begin{array}{ccc}
\boldsymbol{T}_{11} & \cdots & \boldsymbol{T}_{1 r} \\
\vdots & \ddots & \vdots \\
\boldsymbol{T}_{1 r} & \cdots & \boldsymbol{T}_{r r}
\end{array}\right] \leq \mathrm{o} \cdots \cdots \cdots(20)
\end{gathered}
$$

(証明)

リアプノフ関数の候補 $V(\boldsymbol{x}(t))=\boldsymbol{x}^{T}(t) \boldsymbol{P} \boldsymbol{x}(t)$ の差分

$$
\Delta V(\boldsymbol{x}(t))=\boldsymbol{x}^{T}(t+1) \boldsymbol{P} \boldsymbol{x}(t+1)-\boldsymbol{x}^{T}(t) \boldsymbol{P} \boldsymbol{x}(t)
$$

に(16)式を代入すると次のようになる。

$$
\begin{aligned}
\Delta V(\boldsymbol{x}(t))= & \sum_{i=1}^{r} \sum_{j=1}^{r} \sum_{k=1}^{r} \sum_{l=1}^{r} h_{i}(\boldsymbol{z}(t)) h_{j}(\boldsymbol{z}(t)) h_{k}(\boldsymbol{z}(t)) \\
& \times h_{l}(\boldsymbol{z}(t)) \boldsymbol{x}^{T}(t)\left\{( \boldsymbol { A } _ { i } - \boldsymbol { B } _ { i } \boldsymbol { F } _ { j } ) ^ { T } \boldsymbol { P } \left(\boldsymbol{A}_{k}\right.\right. \\
& \left.\left.-\boldsymbol{B}_{k} \boldsymbol{F}_{l}\right)-\boldsymbol{P}\right\} \boldsymbol{x}(t) \\
\leq & \frac{1}{4} \sum_{i=1}^{r} \sum_{j=1}^{r} h_{i}(\boldsymbol{z}(t)) h_{j}(\boldsymbol{z}(t)) \boldsymbol{x}^{T}(t) \\
& \times\left\{\left(\boldsymbol{A}_{i}-\boldsymbol{B}_{\imath} \boldsymbol{F}_{j}+\boldsymbol{A}_{j}-\boldsymbol{B}_{j} \boldsymbol{F}_{i}\right)^{T} \boldsymbol{P}\right. \\
& \left.\times\left(\boldsymbol{A}_{i}-\boldsymbol{B}_{i} \boldsymbol{F}_{j}+\boldsymbol{A}_{j}-\boldsymbol{B}_{j} \boldsymbol{F}_{i}\right)-\boldsymbol{P}\right\} \boldsymbol{x}(t)
\end{aligned}
$$

電学論 C, 120 巻 12 号, 平成 12 年 


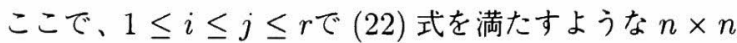
の対称行列 $\boldsymbol{Q}_{i j}=\boldsymbol{Q}_{j i}$ を仮定する。

$$
\begin{aligned}
& \frac{1}{4}\left(\boldsymbol{A}_{i}-\boldsymbol{B}_{i} \boldsymbol{F}_{j}+\boldsymbol{A}_{j}-\boldsymbol{B}_{j} \boldsymbol{F}_{i}\right)^{T} \boldsymbol{P} \\
& \times\left(\boldsymbol{A}_{i}-\boldsymbol{B}_{i} \boldsymbol{F}_{j}+\boldsymbol{A}_{j}-\boldsymbol{B}_{j} \boldsymbol{F}_{i}\right)-\boldsymbol{P}<\boldsymbol{Q}_{i j}, \forall i, j
\end{aligned}
$$

(22) 式より，(21) 式は次のように書きかえられる。

$$
\begin{array}{r}
\Delta V(\boldsymbol{x}(t))<\sum_{i=1}^{r} \sum_{j=1}^{r} h_{i}(\boldsymbol{z}(t)) h_{j}(\boldsymbol{z}(t)) \boldsymbol{x}^{T}(t) \boldsymbol{Q}_{i j} \boldsymbol{x}(t) \\
=\left[h_{1}(\boldsymbol{z}(t)) \boldsymbol{x}^{T}, \cdots, h_{r}(\boldsymbol{z}(t)) \boldsymbol{x}^{T}\right] \boldsymbol{Q} \\
{\left[h_{1}(\boldsymbol{z}(t)) \boldsymbol{x}^{T}, \cdots, h_{r}(\boldsymbol{z}(t)) \boldsymbol{x}^{T}\right]^{T}}
\end{array}
$$

ここで、

$$
\boldsymbol{Q}=\left[\begin{array}{ccc}
\boldsymbol{Q}_{11} & \cdots & \boldsymbol{Q}_{1 r} \\
\vdots & \ddots & \vdots \\
\boldsymbol{Q}_{1 r} & \cdots & \boldsymbol{Q}_{r r}
\end{array}\right]
$$

である。

$\boldsymbol{Q} \leq \mathbf{o}$ が成り立つとき、 $\Delta V(\boldsymbol{x}(t))<0$ となるので、 (16) 式で表される制御系は大域的漸近安定である。

(22) 式に Schur Complement を適用すると次のよう になる。

$$
\left[\begin{array}{cc}
\boldsymbol{P}+\boldsymbol{Q}_{i j} & * \\
\frac{1}{2}\left\{\boldsymbol{A}_{i}-\boldsymbol{B}_{i} \boldsymbol{F}_{j}+\boldsymbol{A}_{j}-\boldsymbol{B}_{j} \boldsymbol{F}_{i}\right\} & \boldsymbol{P}^{-1}
\end{array}\right]>\mathbf{0} \cdots
$$

(24) 式の左右から diag-block $\left[\boldsymbol{P}^{-1}, \boldsymbol{I}\right]$ を掛けると、

$$
\left[\begin{array}{cc}
\boldsymbol{X}+\boldsymbol{T}_{i j} & * \\
\frac{1}{2}\left\{\boldsymbol{A}_{i} \boldsymbol{X}-\boldsymbol{B}_{i} \boldsymbol{M}_{j}+\boldsymbol{A}_{j} \boldsymbol{X}-\boldsymbol{B}_{j} \boldsymbol{M}_{i}\right\} & \boldsymbol{X}
\end{array}\right]>\mathbf{o}(25)
$$

となる。ここで、 $\boldsymbol{X}=\boldsymbol{P}^{-1}, \boldsymbol{M}_{i}=\boldsymbol{F}_{i} \boldsymbol{X}, \boldsymbol{T}_{i j}=$ $\boldsymbol{X} \boldsymbol{Q}_{i j} \boldsymbol{X}$ である。(22),(23) 式より、 $\Delta V(\boldsymbol{x}(t))<0$ と なるための条件は次のようになる。

$\left[\begin{array}{cc}\boldsymbol{X}+\boldsymbol{T}_{i j} & * \\ \frac{1}{2}\left\{\boldsymbol{A}_{i} \boldsymbol{X}-\boldsymbol{B}_{i} \boldsymbol{M}_{j}+\boldsymbol{A}_{j} \boldsymbol{X}-\boldsymbol{B}_{j} \boldsymbol{M}_{i}\right\} & \boldsymbol{X}\end{array}\right]>\mathbf{0}$

$$
i \leq j \cdots(26)
$$

ここで, (20) 式は (23) 式の両辺から $\operatorname{diag}[\boldsymbol{X} \cdots \boldsymbol{X}]$ を掛 けることにより求められる。

[Remark 4.1]

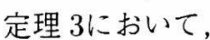

$$
\boldsymbol{T}_{i j}=\left\{\begin{array}{cc}
-(s-1) \boldsymbol{Y} & i=j \\
\boldsymbol{Y} & i \neq j
\end{array}\right.
$$

とおけば定理 3 の条件は定理 2 に帰着する。

\section{5. シミュレーションおよび実験結果}

4 章で導出した安定条件に基づき制御器を設計した。図 3にシミュレーション結果を示す。難しい初期值からでも目
標軌道に追従していることがわかる。

図 4に実験のために作製したモデル機を示す。図 5は実験 装置の概要を示している。制御変数 $x_{6}(t), x_{7}(t)$ は CCD カメラ, 連結部の相対角度 $x_{1}(t), x_{3}(t), x_{5}(t)$ はポテンショ ンメータにより測定する。ステアリング角 $u(t)$ は $(15)$ 式 で表わされるファジィ制御規則から算出する。

ステアリングはステッピングモータで駆動し, 後退は DC モータにより行われる。CCD カメラを含む画像処理システ ムの処理速度の問題からトラック部及び各トレーラ部の位 置を検出するときには多重連結型移動ビークルは停止して おり，制御器により決定されたステアリング角度を切った のち $0.05 \mathrm{~m}$ だけ後退させるようなシステムを組んでいる。

図 6に実験結果を示す。実験においても後退制御が効果 的に実現できることがわかる。実験では，CCD カメラの 視野が狭いという問題から, 難しい初期位值からの制御を 行うことができなかった。今後はこの点を改良していく予 定である。

\section{6. 結 論}

モデルに基づくファジィ制御により多重連結型移動ビー クルの後退制御系を設計した。緩い安定条件に基づく簡易 的な制御系設計法を用いたが，モデル機による実験に成功 した。今㣪はさらに実験を重ね，本手法の有効性と問題点を 明らかにし，それを解決する新たな設計手法の提案を行い たい。本研究に協力いただいた Duke University の Prof. H. Wang 及び電気通信大学の谷口唯成君に感謝する。

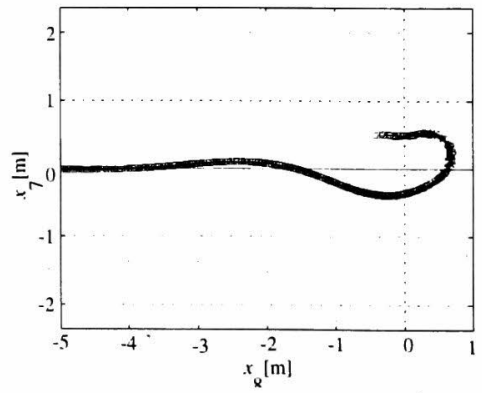

図 3 シミュレーション結果

Fig. 3. Simulation result.

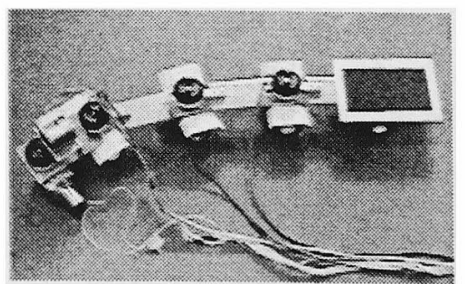

図4 作製したモデル 機の写真

Fig. 4. Vehicle with triple trailers.

(平成 11 年 12 月 7 日受付, 同 12 年 3 月 30 日再受付) 


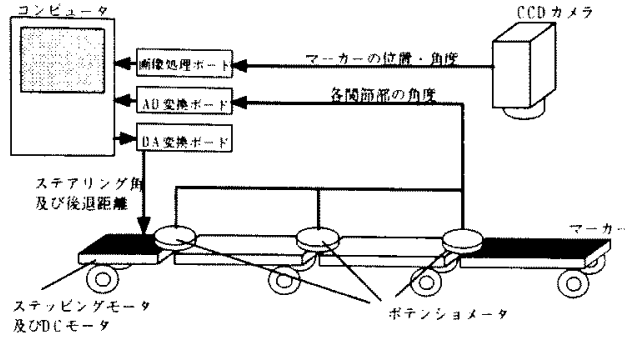

四 5 実験システム

Fig. 5. Experimental system.

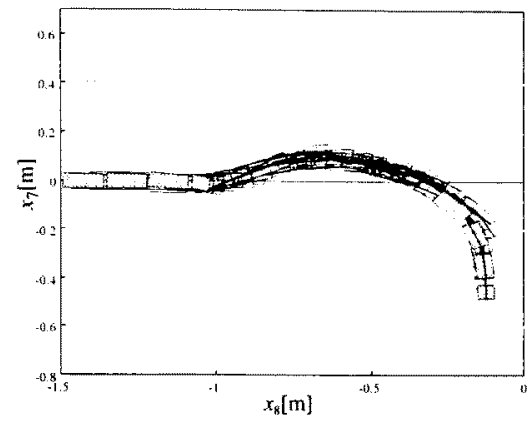

图 6 実験結果

Fig.6. Experimental result.

\section{文献}

(1) D. Nguyen and B. Widrow: "The Truck Backer-upper :An Example of Self-Learning in Neural Networks", Proc. Int Joint Conf. Neural Networks(IJCNN-89), vol. 2, pp. 357363 (1989)

(2) G.S. Kong and B. Kosko: "Adaptive Fuzzy Systems for Backing up a Truck-and-Trailer", IEEE Trans. on Neural Net works, vol. 3 , no. 2 , pp. 211-223 (1992)

(3) H.Inoue, K.Kamei and K.Inoue: "Auto-Generation of Fuzzy Production Rules Using Hyper-Cone Membership Function by Genetic Algorithm", Proc. Int. Joint Conf CFSA/IFIS/SOFT'95, pp. 53-58 (1995)

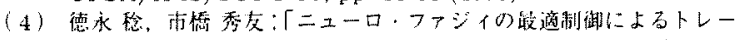

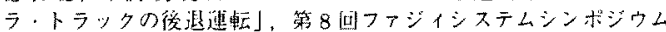
(広島, May 26-28) pp.49-52 (1992)

(5) K.Tanaka and T. Kosaki: "Design of a Stable Fuzzy Controller for an Articulated Vehicle", IEEE Transactions on Systems, Man and Cybernetics : Part B, Vol. 27, No. 3, pp 552-558 (1997)

(6) M. Sampei, et al., "Arbitrary Path Tracking Control of Articulated Vehicles Using Nonlinear Control Theory", IEEE Trans. on Control Systems Technology. vol.3, no.1, pp.125131 (1995)

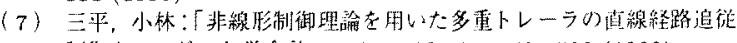
制御」， 口ボット学丟婄，Vol.11，No.4，pp587-592 (1993)

(8) M. Sampei, T. Kobayashi, Y. Tajima, N. Shibui and M Ishikawa: "Application of Nonlinear Control Theory to Path Tracking Control of Articulated Vehicles", 1995 IEEE International Conference on Robotics and Automation, Video Proceedings (1995)

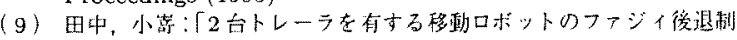

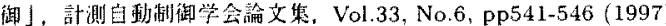

(10) K. Tanaka and M. Sugeno: "Stability Analysis of Fuzzy Systems Using Lyapunov's Direct Method", Proc. of NAFIPS'90, pp. 133-136 (1990)

(11) K. Tanaka, and M.Sugeno: "Stability Analysis and Design of Fuzzy Control Systems", FUZZY SETS AND SYSTEMS, vol. 45 , no. 2, pp. 135-156 (1992)

(12) H. O. Wang, K. Tanaka and M. Griffin: "Parallel Distributed Compensation of Fuzzy Systems via Takagi-Sugeno Fuzzy Model", Proc. of 4th IEEE Int. Conf. on Fuzzy Systems, vol. 2, pp. $531-538$ (1995)

(13) H. O. Wang, K. Tanaka and M. Griffin: "An Approach to Fuzzy Control of Nonlinear Systems", IEEE Transactions on Fuzzy Systems, vol. 4, No. 1, pp. 14-23 (1996)

(14) K.Tanaka, T. Ikeda and H. O. Wang: "Fuzzy Regulators and Fuzzy Observers: Relaxed Stability Conditions and LMI based Designs", IEEE Trans. on Fuzzy Systems, vol.6, No. 2, pp. 250-265 (1998)

(15) T. Takagi and M.Sugeno: "Fuzzy Identification of Systems and Its Applications to Modeling and Control", IEEE SMC, vol. 15, no. 1, pp. 116-132 (1985)

(16) S.Boyd et.al.: "Linear Matrix Inequalities in Systems and Control Theory", SIAM, Philadelphia, (1994).

(17) Yu.Nesterov and A.Nemirovsky: "Interior-point polynomial methods in convex programming", SIAM, Philadelphia, PA(1994).

(18) K. Tanaka and H. O. Wang, "Fuzzy Control Systems and Analysis: A Linear Matrix Inequality Approach", John Wi ley Ef Sons, $(2000)$.

\section{付 録}

\section{1. ファジィモデル化}

実験での後退制御の成功を第一の目的としているため，非 線形方程式 $(1)$ （8) 式をできるたけ簡単化し，制㣤系設計 を行う。簡単化したモデルで設計した制御器がうまく動か ないときはより詳細なモデルを考える方針であったが，本 付録に示すような簡単なファジィモデルにより設計した制 御器で㖟退制御を行うことができた。実験による経験から $u(t)$ と $x_{1}(t), x_{3}(t), x_{5}(t)$ の值が小さくないと捘退制御は 成功に至らない。そこで, $u(t), x_{1}(t), x_{3}(t), x_{5}(t)$ が小さ い值であるという仮定を考えると，(1)一(8) 式から次の簡 単化システムを得る。

$x_{0}(t+1)=x_{0}(t)+\frac{\nu \cdot \Delta t}{\ell} u(t)$

$x_{1}(t+1)=\left(1-\frac{\nu \cdot \Delta t}{L}\right) x_{1}(t)+\frac{\nu \cdot \Delta t}{\ell} u(t)$

$x_{2}(t+1)=x_{2}(t)+\frac{\nu \cdot \Delta t}{L} x_{1}(t)$

$x_{3}(t+1)=\left(1-\frac{\nu \cdot \Delta t}{L}\right) x_{3}(t)+\frac{\nu \cdot \Delta t}{L} x_{1}(t)$

$x_{4}(t+1)=x_{4}(t)+\frac{\nu \cdot \Delta t}{L} x_{3}(t)$.

$x_{5}(t+1)=\left(1-\frac{\nu \cdot \Delta t}{L}\right) x_{5}(t)+\frac{\nu \cdot \Delta t}{L} x_{3}(t)$

$x_{6}(t+1)=x_{6}(t)+\frac{\nu \cdot \Delta t}{L} x_{5}(t)$

$\left.x_{7}(t+1)=x_{7}(t)+\nu \cdot \Delta t \sin \left(x_{6}(t)\right)+\frac{\nu \cdot \Delta t}{2 L} x_{5}(t)\right)$

(付 8) 式注非線形項

$$
\nu \cdot \Delta t \cdot \sin \left(x_{6}(t)+\frac{\nu \cdot \Delta t}{2 L} x_{5}(t)\right)
$$

を持つ。文献 (5),(9)のモデル化と同様 $\sin$ 関数を sector nonlinearity ${ }^{(18)}$ の考え方でモデル化を行うと(付 8) 式の 非線形項は次の高木・菅野のファジィモデル形式で表現で きる。 


$$
\begin{aligned}
& \nu \cdot \Delta t \cdot \sin \left(x_{6}(t)+\frac{\nu \cdot \Delta t}{2 L} x_{5}(t)\right) \\
& =w_{1}(p(t)) \cdot \nu \cdot \Delta t \cdot\left(x_{6}(t)+\frac{\nu \cdot \Delta t}{2 L} x_{5}(t)\right) \\
& +w_{2}(p(t)) \cdot \nu \cdot \Delta t \cdot g \cdot\left(x_{6}(t)+\frac{\nu \cdot \Delta t}{2 L} x_{5}(t)\right) \text {. (付 10) } \\
& \text { ここで } \\
& p(t)=x_{6}(t)+\frac{\nu \cdot \Delta t}{2 L} x_{5}(t), \quad g=10^{-2} / \pi, \\
& w_{1}(p(t))=\left\{\begin{array}{cc}
\frac{\sin (p(t))-g \cdot p(t)}{p(t) \cdot(1-g)}, & p(t) \neq 0 \\
1 & p(t)=0
\end{array}\right. \\
& w_{2}(p(t))=\left\{\begin{array}{cc}
\frac{p(t)-g \cdot \sin (p(t))}{p(t) \cdot(1-g)}, & p(t) \neq 0 \\
1 & p(t)=0
\end{array}\right.
\end{aligned}
$$

である。(付 11)，(付 12) 式より" $p(t)$ is about 0 [rad.]"の とき, $w_{1}(p(t))=1, w_{2}(p(t))=0, " p(t)$ is about $\pi$ or - $\pi$ [rad.]" のとき, $w_{1}(p(t))=0, w_{2}(p(t))=1$ となる。 $w_{1}(p(t))=1, w_{2}(p(t))=0$, すなわち " $p(t)$ is about 0 [rad.]”のとき（付 10) 式を(付 8 ) 式に代入すると次式を 得る。

$x_{7}(t+1)=x_{7}(t)+\nu \cdot \Delta t \cdot x_{6}(t)+\frac{(\nu \cdot \Delta t)^{2}}{2 L} \cdot x_{5}(t)$

したがって，この状況では以下のようなモデルが得られる。

$$
\left[\begin{array}{l}
x_{1}(t+1) \\
x_{3}(t+1) \\
x_{5}(t+1) \\
x_{6}(t+1) \\
x_{7}(t+1)
\end{array}\right]
$$$$
=\left[\begin{array}{ccccc}
1-\frac{\nu \cdot \Delta t}{L} & 0 & 0 & 0 & 0 \\
\frac{\nu \cdot \Delta t}{L} & 1-\frac{\nu \cdot \Delta t}{L} & 0 & 0 & 0 \\
0 & \frac{\nu \cdot \Delta t}{L} & 1-\frac{\nu \cdot \Delta t}{L} & 0 & 0 \\
0 & 0 & \frac{\nu \cdot \Delta t}{L} & 1 & 0 \\
0 & 0 & \frac{(\nu \cdot \Delta t)^{2}}{2 L} & \nu \cdot \Delta t & 1
\end{array}\right]\left[\begin{array}{l}
x_{1}(t) \\
x_{3}(t) \\
x_{5}(t) \\
x_{6}(t) \\
x_{7}(t)
\end{array}\right]
$$$$
+\left[\begin{array}{c}
\frac{\nu \cdot \Delta t}{\ell} \\
0 \\
0 \\
0 \\
0
\end{array}\right] u(t)
$$

一方, $w_{1}(p(t))=0, w_{2}(p(t))=1$, すなわち" $p(t)$ is about $\pi$ or $-\pi[\mathrm{deg}]$.$" のとき，(付 8)$ 式は次のように変形できる。

$x_{7}(t+1)=x_{7}(t)+g \cdot \nu \cdot \Delta t \cdot x_{6}(t)+\frac{g \cdot(\nu \cdot \Delta t)^{2}}{2 L} \cdot x_{5}(t)$

したがって，この状況では以下のようなモデルが得られる。

$$
\left[\begin{array}{l}
x_{1}(t+1) \\
x_{3}(t+1) \\
x_{5}(t+1) \\
x_{6}(t+1) \\
x_{7}(t+1)
\end{array}\right]
$$

$$
\begin{aligned}
& =\left[\begin{array}{cccrr}
1-\frac{\nu \cdot \Delta t}{L} & 0 & 0 & 0 & 0 \\
\frac{\nu \cdot \Delta t}{L} & 1-\frac{\nu \cdot \Delta t}{L} & 0 & 0 & 0 \\
0 & \frac{\nu \cdot \Delta t}{L} & 1-\frac{\nu \cdot \Delta t}{L} & 0 & 0 \\
0 & 0 & \frac{\nu \cdot \Delta t}{L} & 1 & 0 \\
0 & 0 & \frac{g \cdot(\nu \cdot \Delta t)^{2}}{2 L} g \cdot \nu \cdot \Delta t 1
\end{array}\right]\left[\begin{array}{l}
x_{1}(t) \\
x_{3}(t) \\
x_{5}(t) \\
x_{6}(t) \\
x_{7}(t)
\end{array}\right] \\
& +\left[\begin{array}{c}
\frac{\nu \cdot \Delta t}{\ell} \\
0 \\
0 \\
0
\end{array}\right] u(t) \ldots \ldots \ldots \ldots \ldots \ldots \ldots \text { (付 14) }
\end{aligned}
$$

この表現では $g=0$ のとき (付 14 ) 式は不可制御となる。 この問題を解消するために $g=10^{-2} / \pi$ とする。このよう にgをとったことにより, (付 9) 式の非線形項は

$$
-179.4270[\text { deg. }]<p(t)<179.4270[\text { deg. }]
$$

の範囲において (付 10) 式のように近似できる。(付 13), (付 14) 式より (10) 式の高木・菅野のファジィモデルを得る。

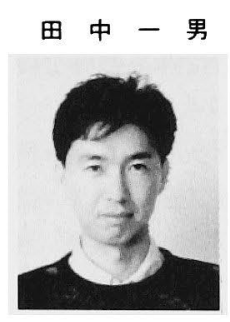

(正員) 1962 年 11 月 22 日生まれ. 1990 年, 東 京工業大学総合理工学研究科システム科学専攻 博士後期課程修了. 工学博士. 同年金沢大学工学 部機械システム工学科助手, 1994 年同学科助教 授, 1996 年人間 ·機械工学科助教授, 1998 年電 気通信大学機械制御工学科助教授、現在に至る. 1992 年 University of North Carolina 客員研究 員. NAFIPS'90、HJSFSA'91 にて優秀論文賞， 日本機械学会賞奖励賞、日本ファジィ学会奨励賞および著述賞、1999 年 IFAC World Congress Best Poster Paper Prize、2000 IEEE Transactions on Fuzzy Systems Outstanding Paper Award などを 受賞、知的制御システムの解析と設計およびその応用に関する研究に 従事.

堀

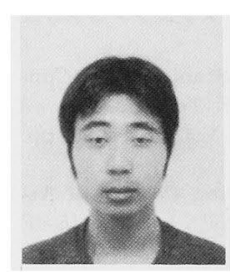

強（非会員） 1977 年 1 月 24 日生まれ。 1999 年 3 月 電気通信大学機械制御工学科卒業. 同年 4 月同大 学電気通信学研究科機械制御工学専攻博士前期課 程入学. 現在に至る.ファジィ制御采の解析と設 計に関する研究に従事.

岩 崎 正 明（非会員） 1977 年 12 月 6 日生まれ。1996 年 4 月 電気通信大学機械制御工学科入学. 現在に至る. 知的制御システムの応用に関する研究に従事.

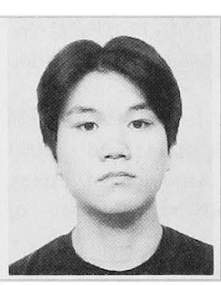

